\title{
Guías para cirugía refractiva con láser excimer, femtosegundos y tratamientos de cross linking en pandemia de COVID-19 en México
}

\section{Guidelines for refractive surgery with excimer and femtosecond lasers and for treatment with cross-linking during the COVID-19 pandemic in Mexico}

\author{
Oscar Fernández-Vizcaya ${ }^{1 *}$, Alejandro Babayán-Sosa², Regina Velasco-Ramos \\ Julia C. Pacheco-del-Valle", Elisa Alegría-Gómez ${ }^{1}$ y Oscar Baca-Lozada ${ }^{1}$ \\ ${ }^{1}$ Departamento de Córnea y Cirugía Refractiva; ${ }^{2}$ Subdirección Médica. Fundación Hospital Nuestra Señora de la Luz, Ciudad de México, México
}

\section{Resumen}

La grave pandemia causada por el coronavirus respiratorio SARS-CoV-2 (COVID-19) es actualmente un problema muy importante en todas las clínicas de cirugías electivas, incluida la cirugía refractiva, las cuales han reprogramado la mayoría de sus procedimientos por un tiempo prolongado debido a la alta morbilidad y mortalidad. Actualmente, gracias a los cambios en el conocimiento de la enfermedad, así como a los cuidados con barreras de protección, muchas clínicas de cirugía refractiva han reiniciado sus procedimientos sin contar aún con un consenso en cuanto a protección para médicos y pacientes. Este trabajo pretende adaptar la información internacional de las guías existentes, así como aportar recomendaciones que puedan aplicarse y ser de utilidad para la práctica de estos procedimientos en México.

Palabras clave: COVID19. Prácticas preferidas. Cirugía refractiva. Guías.

\begin{abstract}
The severe pandemic caused by the respiratory coronavirus SARS-CoV-2 (COVID-19) is currently a major problem in all elective surgery clinics, including those that perform refractive surgery, which has been rescheduling most of their procedures for a long time due to high morbidity and mortality. Currently, due to changes in the knowledge of the disease, as well as protective measures with barriers, many refractive surgery clinics have restarted their procedures without yet having a consensus about protection for doctors and patients. This work aims to adapt the international information of the existing guidelines, as well as to provide recommendations that can be applied and be useful for the practice of these procedures in Mexico.
\end{abstract}

Key words: COVID19. Preferred practice. Refractive surgery. Guidelines.

Correspondencia:

*Oscar Fernández-Vizcaya

Ezequiel Montes 135

Col. Tabacalera, Del. Cuauhtémoc

Fecha de recepción: 13-11-2020

C.P. 06030, Ciudad de México, México

E-mail: droscarfdez@gmail.com

0187-4519/○ 2021 Sociedad Mexicana de Oftalmología. Publicado por Permanyer. Este es un artículo open access bajo la licencia CC BY-NC-ND (http://creativecommons.org/licenses/by-nc-nd/4.0/).
Disponible en internet: 01-07-2021 Rev Mex Oftalmol. 2021;95(4):161-166

www.rmo.com.mx
Fecha de aceptación: 06-04-2021 


\section{Introducción}

La COVID-19, causada por un coronavirus descubierto en China a finales de 2019 y que ha ocasionado una pandemia con más de 800,000 muertos hasta septiembre de $2020^{1}$, tuvo su primer caso confirmado en México el 27 de febrero de 2020, con un aumento significativo de los casos a lo largo de los meses, estando actualmente entre los países con mayor número de casos y mayor mortalidad².

El mecanismo de transmisión que hasta la fecha se ha establecido es principalmente a través de microgotas generadas cuando una persona infectada tose, estornuda o habla, y estas entran en contacto de manera directa con las mucosas de las vías respiratorias altas, o de manera indirecta por medio de fómites. Además de estos mecanismos, se cree que otra vía de transmisión puede ser aérea en lugares cerrados y con hacinamiento; aunado a esto, el aparato respiratorio no debe considerarse la única vía de transmisión, pues las lágrimas y la ruta oral-fecal son otro potencial portal de entrada ${ }^{3}$. Las conjuntivitis no son una manifestación frecuente de la enfermedad, pero el contacto con los ojos infectados podría ser una vía de transmisión, y como consecuencia, los oftalmólogos deben tener estrategias de prevención para la práctica, tanto en el consultorio como en el quirófano, para todos los tipos de cirugía, incluyendo la refractiva ${ }^{4}$. Aunque la posibilidad de contagio por vía de la superficie ocular se cree baja, se han documentado receptores de la enzima convertidora de la angiotensina 2 en la superficie del ojo $^{5}$, así como células conjuntivales infectadas en cultivos humanos ex vivo ${ }^{6}$. En las lágrimas es difícil estimar el desprendimiento viral, ya que el periodo de ventana puede durar un tiempo corto 7 , y por lo tanto, durante los procedimientos refractivos láser y de cross linking en los que el oftalmólogo tiene una distancia reducida con los pacientes, pudiera entrar en contacto con lágrimas que se dispersaran en forma de gotas 0 aerosoles, y con humo quirúrgico con micropartículas producido por los láseres, por lo que a continuación se establecen algunas guías y recomendaciones para la prevención durante la consulta oftalmológica y la cirugía refractiva.

Se presentan a continuación las medidas que pueden tomarse en hospitales y clínicas donde se realiza cirugía refractiva y cross linking, de acuerdo con las recomendaciones de la literatura y lo observado en la práctica.

\section{Medidas generales para las clínicas y hospitales donde se realizan procedimientos refractivos}

\section{Prehospitalario}

- Control del número y programación de citas por teléfono para evitar la saturación de pacientes y un mayor tiempo de permanencia en el hospital.

- Interrogar por vía telefónica sobre la sintomatología de los pacientes a ingresar a la clínica, considerar cuestionarios online que incluyan preguntas sobre viajes, fiebre, tos, dificultad reciente para respirar, pérdida del olfato o del gusto, tanto para pacientes como para acompañantes.

- Gestión de control de espacios y salas de espera en el hospital para mantener una distancia mínima de 2 $m$ entre los pacientes

- Informar a los pacientes sobre los métodos de pago en línea para reducir el tiempo de estancia en el hospital y minimizar el contacto con billetes y monedas.

\section{En el hospital}

\section{PaRA PACIENTES}

- Cubrebocas obligatorio para pacientes y familiares.

- Entrada solo de acompañantes indispensables en caso de problema de movilidad del paciente 0 acompañante de cirugía (solo permitir la entrada a un familiar por procedimiento).

- Toma de temperatura mediante pistola de infrarrojos a todo paciente y acompañante que ingresan al hospital.

- Gel antibacteriano, si es posible en dispensador automático para evitar el contacto, en la entrada y en varios puntos estratégicos de la clínica 0 del hospital.

- Distanciamiento social preferiblemente mayor de $2 \mathrm{~m}$ en todas las áreas, como farmacia, laboratorios, recepción, áreas de movilidad y zonas de espera.

- Zona de control de síntomas, donde se realizan oximetría de pulso, cuestionario de síntomas y nueva toma de temperatura.

- Proporcionar tanto al paciente como al acompañante los datos de la aplicación de COVID 19MX de la Secretaría de Salud para acceso al teléfono de atención epidemiológica sanitaria, autodiagnóstico y ubicación de los centros de atención. 


\section{ZONAS DE CONSULTORIOS}

- Protección de las lámparas de hendidura mediante cobertura plástica entre paciente y médico, solicitando a los pacientes no hablar durante la revisión en la lámpara de hendidura (Fig. 1).

- Lavado de manos antes de tocar al paciente, antes de realizar una tarea limpia/aséptica, después del riesgo de exposición a líquidos corporales, después de tocar al paciente y después del contacto con el entorno del paciente.

- Desinfección de los tonómetros con alcohol al 70\% ${ }^{8}$, esperando al menos 1 minuto antes de su uso, o utilización de tonómetro con cobertura desechable, como Tono-Pen®.

- Reducción de los módulos de atención de consulta para ampliar la distancia entre pacientes y médicos a $2 \mathrm{~m}$.

\section{SALAS DE JUNTAS INTRAHOSPITALARIAS Y OFICINAS DE DEPARTAMENTOS DE ESPECIALIDAD}

- Mantener siempre abiertas por ventilación.

- Mínimo personal y a más de 2 m entre ellos.

- Desinfección constante de superficies y equipos dentro de ellas.

\section{ZONA DE TOPOGRAFÍA}

- Limitar el ingreso de pacientes para estudios de topografía y tomografía a un paciente por estudio.

- En caso de utilizar otro instrumento, como ultrasonido, tomografía de coherencia óptica o microscopio confocal, solo podrá ingresar un paciente y respetando el espaciamiento mayor de $2 \mathrm{~m}$ entre cada aparato, así como entre pacientes, médicos y técnicos.

- Dada la dificultad de colocar escudos plásticos en el topógrafo, el técnico que realiza la topografía debe portar, además de cubrebocas, un protector de acetato plástico para la cara.

\section{ZONA DE REFRACCIÓN}

- Desinfección de foroptor y autorrefractómetro con alcohol al $70 \% 8$.

- Protectores acrílicos en foroptor para disminuir los aerosoles al refractar.

- Para cicloplejía, resguardo de gotas multiuso entre pacientes para evitar la contaminación de estas ${ }^{9}$.

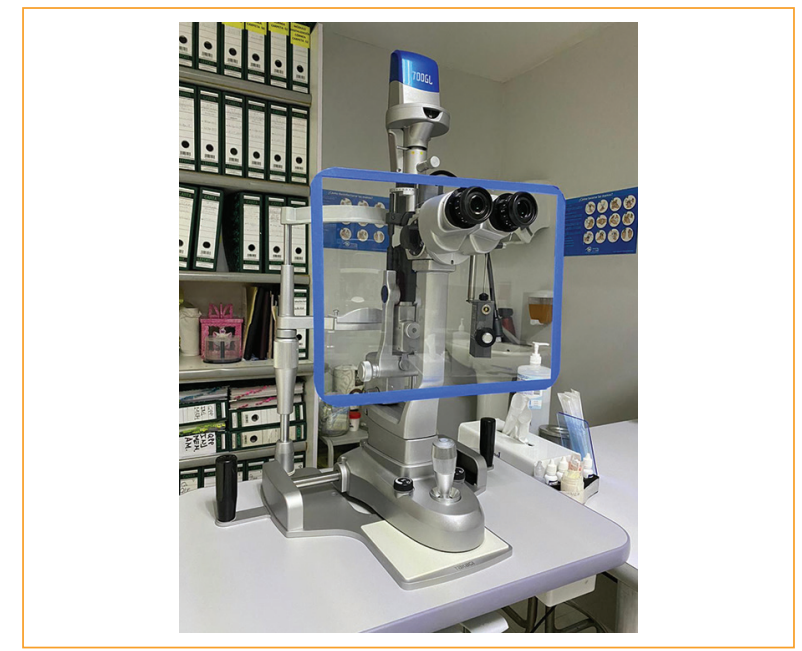

Figura 1. Separador plástico para lámpara de hendidura, fácil de hacer y adaptar para los diferentes tipos de lámparas.

\section{Para empleados del hospital}

- Todo el personal involucrado debe tener cubrebocas, gafas protectoras, gorro para la cabeza y guantes en todo momento. Cada hospital o clínica puede establecer guías informativas sobre barreras de protección, para hacer disponible esta información a todos sus empleados.

- Uso de gel o lavado de manos antes y después de entrar en contacto con objetos del paciente.

- Saludar a los pacientes manteniendo la distancia recomendada en lugar de dar la mano.

- Mantener puertas y ventanas abiertas para permitir el flujo libre de aire.

- Limpiar todas las superficies (unidades de sillas, mesas, teclados, monitores de computadora) con soluciones desinfectantes a intervalos periódicos.

\section{Programación de cirugía}

- Examinador con cubrebocas N95 y gafas de protección, y paciente con cubrebocas obligatorio.

- Oclusores con agujero estenopeico, así como mentonera y apoyo para la frente del autorrefractómetro, desinfectados con alcohol al $70 \%$ entre cada paciente ${ }^{8}$.

- Durante la refracción, evitar apoyarse en el paciente al tomar la distancia de trabajo y no hablar durante la evaluación.

- Utilizar autorrefractómetro durante la revisión para complementar y disminuir el tiempo en el foroptor, el cual debe contar con una barrera plástica.

- Desinfectar el apoyo para el mentón y la frente de la lámpara de hendidura con alcohol al $70 \%$ entre cada 
paciente, y esperar por lo menos 1 minuto antes de volver a utilizarlo ${ }^{9}$.

- El prisma del tonómetro Goldman de la lámpara, además de la desinfección con alcohol, debe ser desinfectado con hipoclorito de sodio 1:10 al inicio y al final de las consultas de cada día.

- En caso de utilizar un tonómetro de aire será importante colocar una protección de barrera entre el paciente y el médico.

- Desinfectar entre pacientes el mango del retinoscopio y la zona plástica, evitando tocar el espejo, con alcohol al $70 \%$.

- El consentimiento firmado para cirugía deberá contar con un anexo que contenga información sobre la COVID-19 y el riesgo de contagio.

\section{Preoperatorio}

- El día del procedimiento se deberán realizar todos los pasos ya descritos previamente a la llegada al hospital.

- Se verifican el cuestionario de síntomas y contactos con enfermos de COVID-19 por escrito y la firma del paciente.

- En hospitalización para el paciente:

- Cambio de cubrebocas.

- Toma de temperatura, signos vitales y saturación de oxígeno con oxímetro de pulso.

- Proporcionar gorro, pijama y botas quirúrgicas desechables.

\section{En el área general de quirófanos y las zonas de tránsito entre ellos}

- Uso de equipo de protección para el personal médico de acuerdo con las recomendaciones generales de quirófano, con bata, guantes y gorro, además de lentes o gafas de protección.

- Doble cubrebocas, uno N95 y otro tricapa quirúrgico sobre este.

- Pijama quirúrgico de material desechable tanto para personal médico, de enfermería y camilleros como para pacientes, para evitar fómites.

- Obligatorio para el paciente el uso de cubrebocas, de preferencia tipo N95.

\section{Precauciones intraoperatorias del quirófano refractivo para excimer y cross linking}

- Aplicación de povidona yodada en fondo de saco, al $0.25 \%$, 10 minutos antes de la cirugía. Esto es viricida y afecta de manera mínima la superficie ocular $^{10}$.

Asegurar la formación de una cámara de separación para evitar la dispersión de aerosoles por el microqueratomo o la irrigación de la superficie ocular, así como disminuir la dispersión de las partículas del humo quirúrgico y la respiración de paciente y cirujano, utilizando una bolsa plástica transparente de polipropileno que puede esterilizarse mediante óxido de etileno, entre paciente y cirujano, cortada previamente para adaptarse a la forma de cada equipo de

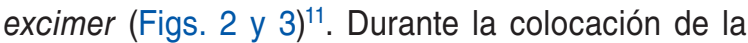
barrera plástica es indispensable no ocluir las zonas de ventilación del excimer para evitar un mal funcionamiento (Fig. 4).

- Cubrir perfectamente al paciente con campos estériles desechables.

- Asepsia de la cara y las manos del paciente.

- Explicación del procedimiento, aclaración de dudas y comentarios antes de ingresar a quirófano. Dentro del quirófano, prohibido hablar.

- El médico (Fig. 5):

- Gafas de protección, cubrebocas KN95 y cubrebocas triple capa.

- Uniforme quirúrgico y bata.

- Lavado de manos habitual.

- Gorro que asegure la cobertura total del cabello.

- Lavado de manos posterior al procedimiento quirúrgico.

- Retiro del uniforme quirúrgico en orden específico.

- Cambiar bata y realizar lavado entre cada paciente.

- Disponer de varias cajas de equipo esterilizadas para no reutilizar instrumentales y materiales.

- Mantener una distancia constante entre los asistentes del quirófano.

- Preferir utilizar el monitor para la visualización si es posible durante el corte con microqueratomo para evitar aerosoles, así como mientras se dispara con el láser, para reducir, junto con el extractor de aire, la exposición a las partículas generadas por el láser $^{12}$.

- Evitar una irrigación excesiva de la interfase para no generar un acúmulo de las soluciones con la lágrima y considerar que dicha irrigación puede generar aerosoles ${ }^{11}$.

- Al terminar el procedimiento, realizar una limpieza de la cama del paciente con desinfectantes. Los pisos circundantes deben limpiarse y dejarse secar después de la cirugía. 


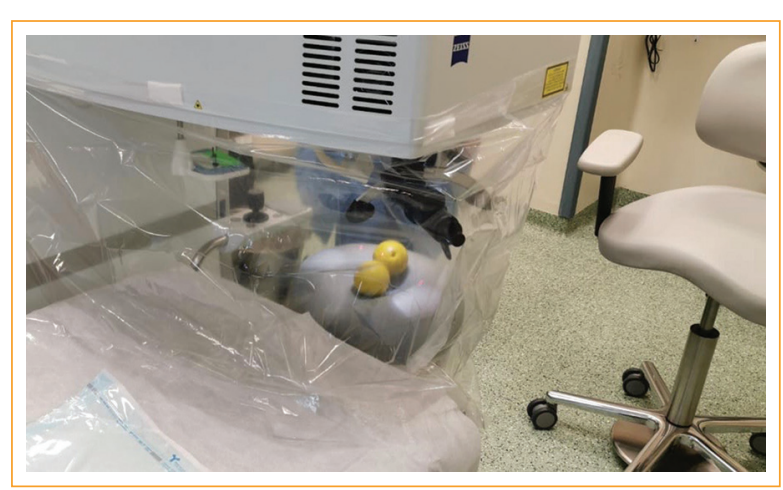

Figura 2. Barrera plástica esterilizada para cumplir la función de cámara de aislamiento durante la aplicación del láser excimer.

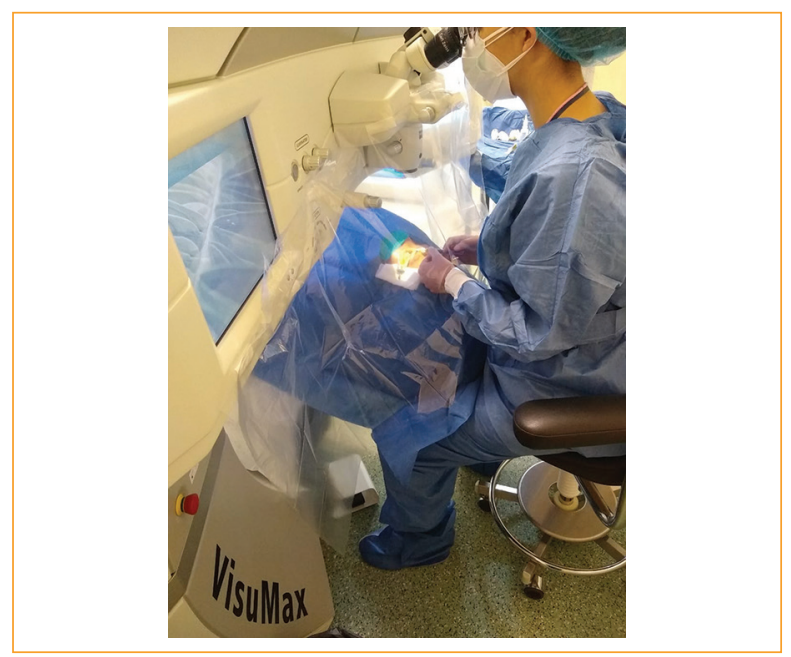

Figura 3. Barrera plástica colocada en el láser de femtosegundos, la cual se aprovecha para la desepitelización durante el cross linking previo a la aplicación de luz ultravioleta.

- Al realizar el aseo del área, cuidar de no rociar los productos desinfectantes sobre los lentes del excimer, y realizar su limpieza entre cada paciente de acuerdo con el manual de limpieza y mantenimiento de cada excimer específico.

- En caso de haber utilizado láser de femtosegundos, desechar los materiales utilizados para cada paciente, como pueden ser la copa de succión y los tubos, dependiendo de cada sistema de láser.

- En caso de cross linking para desepitelizar puede utilizarse el microscopio del láser con el mismo sistema plástico de separación entre paciente y cirujano.

- Considerar, tanto para PRK como para cross linking, colocar la barrera plástica entre cirujano y paciente

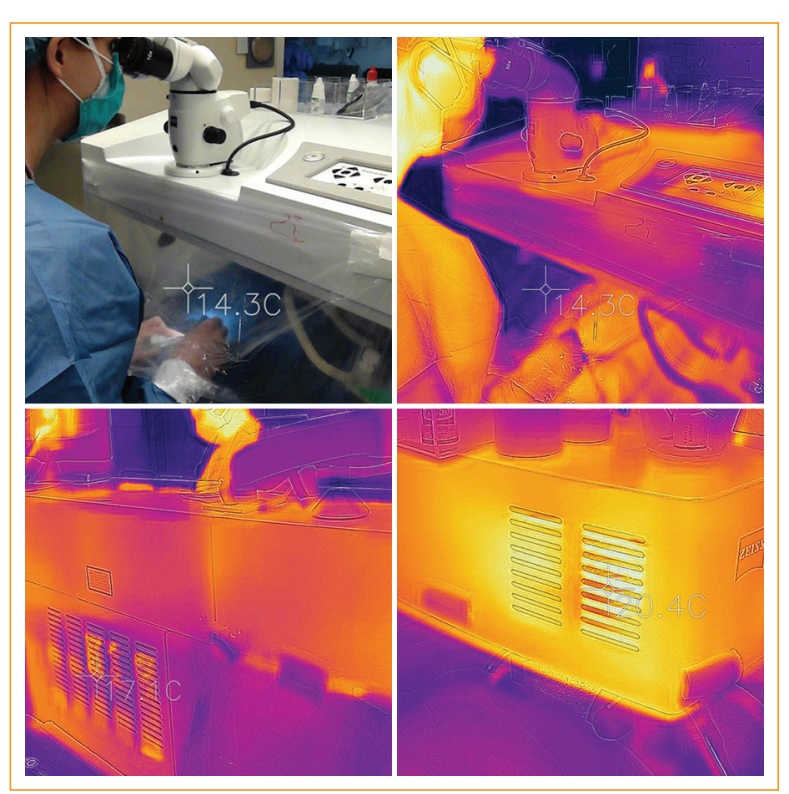

Figura 4. Imágenes termográficas que muestran en los dos cuadros superiores el interior de la barrera plástica donde se encuentra el paciente mediante una cámara termográfica Flir One Pro (resolución térmica $160 \times 120$, resolución óptica $1440 \times 1080$ ), evidenciando que no se eleva de manera importante dado que no son completamente herméticas, pero cumplen la función de reducir las gotas generadas por los microqueratomos. En los dos cuadros inferiores se aprecian las zonas de ventilación del excimer sin ocluir con la barrera ya colocada para no afectar la temperatura del equipo.

dado los aerosoles generados por la irrigación para retirar el alcohol diluido durante la desepitelización.

- En cross linking cubrir toda la parte plástica de la lámpara, excepto el área de la lente que administra la luz ultravioleta, con un plástico estéril desechable, el cual se deberá cambiar entre pacientes.

- Al terminar el día quirúrgico, además de la limpieza general del quirófano, si es posible mantener una luz ultravioleta prendida durante la noche ${ }^{13}$.

\section{Cuidados posoperatorios}

Además de los cuidados habituales, considerar reducir las visitas posoperatorias al mínimo indispensable, con la posibilidad de videoconsulta para dudas no urgentes.

Mantener las medidas del preoperatorio de distanciamiento y limpieza de los equipos, así como los cuidados del personal médico y otros empleados de la clínica u hospital.

Todas las intervenciones anteriores son propensas a fallas y, por lo tanto, requieren retroalimentación de 


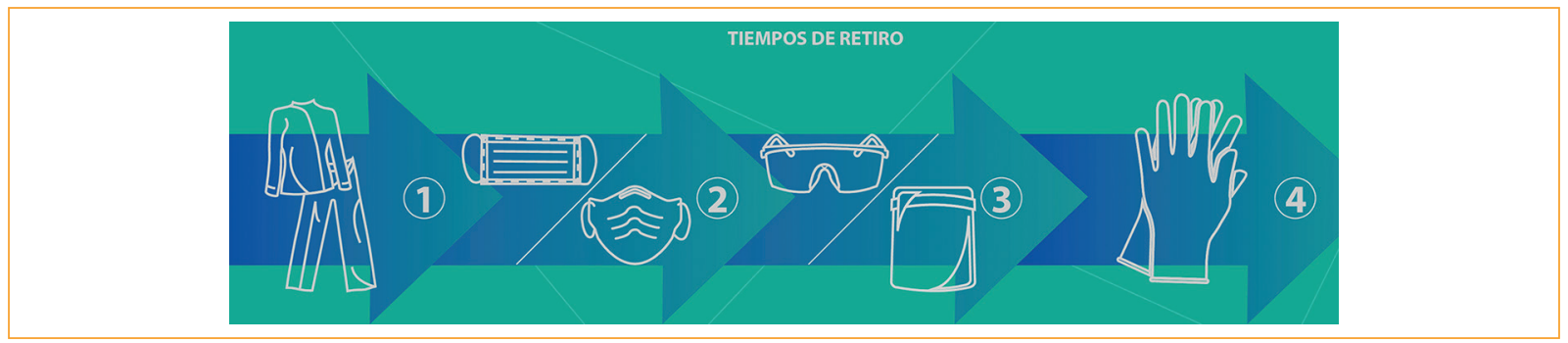

Figura 5. Secuencia de retiro del uniforme quirúrgico.

datos para mantener la fidelidad, por lo que es indispensable reportar cualquier error cometido.

Es conveniente realizar el seguimiento del paciente y su acompañante por vía telefónica, cuestionarios o aplicaciones gratuitas para sistemas Android o IOS.

\section{Conclusiones}

Aunque la cirugía refractiva es un procedimiento electivo, es indispensable plantear la recuperación gradual de estas cirugías y consultas en oftalmología para las diferentes especialidades. La elaboración de guías dinámicas, que puedan ser modificadas según el conocimiento actualizado y adaptado para cada país, hará posible la opción de reducir los riesgos tanto para los oftalmólogos y el personal que labora en clínicas y hospitales como para los pacientes, de quienes además es indispensable recuperar gradualmente la confianza para estos procedimientos.

\section{Financiación}

Los autores declaran que el estudio no recibió ningún tipo de financiación o aportación económica.

\section{Conflicto de intereses}

Los autores declaran no tener ningún conflicto de intereses.

\section{Responsabilidades éticas}

Protección de personas y animales. Los autores declaran que para esta investigación no se han realizado experimentos en seres humanos ni en animales.
Confidencialidad de los datos. Los autores declaran que en este artículo no aparecen datos de pacientes.

Derecho a la privacidad y consentimiento informado. Los autores declaran que en este artículo no aparecen datos de pacientes.

\section{Bibliografía}

1. COVID-19 Dashboard by the Center for Systems Science and Engineering (CSSE) at Johns Hopkins University (JHU). ArcGIS. Johns Hopkins University. (Consultado el 14 de julio de 2020.) Disponible en: https:// systems.jhu.edu/research/public-health/ncov/.

2. Datos abiertos. Dirección General de Epidemiología. Secretaría de Salud, Gobierno de México. Disponible en: https://datos.gob.mx/busca/dataset/informacion-referente-a-casos-covid-19-en-mexico.

3. Godri Pollitt KJ, Peccia J, Ko Al, Kaminski N, Dela Cruz CS, Nebert DW et al. COVID-19 vulnerability: the potential impact of genetic susceptibility and airborne transmission. Hum Genomics. 2020;14:17.

4. Amesty MA, Alió Del Barrio JL, Alió JL. COVID-19 disease and ophthalmology: an update. Ophthalmol Ther. 2020;9:1-12.

5. Zhou L, Xu Z, Castiglione GM, Soiberman US, Eberhart CG, Duh EJ. ACE2 and TMPRSS2 are expressed on the human ocular surface, suggesting susceptibility to SARS-CoV-2 infection. Ocul Surf. 2020;18:537-44.

6. Hui KPY, Cheung MC, Perera RAPM, Ng KC, Bui CHT, Ho JCW, et al. Tropism, replication competence, and innate immune responses of the coronavirus SARS-CoV-2 in human respiratory tract and conjunctiva: an analysis in ex-vivo and in-vitro cultures. Lancet Respir Med. 2020;8: 687-95.

7. Hamre D, Procknow JJ. A new virus isolated from the human respiratory tract. Proc Soc Exp Biol Med. 1966;121:190-3.

8. Centers for Disease Control and Prevention. COVID-19: resources for households, 2020. Disponible en: https://www.cdc.gov/coronavirus/2019-ncov/prepare/cleaning-disinfection.html?CDC_AA_refVal=https\%3A\%2F\%2Fwww.cdc.gov\%2Fcoronavirus\%2F2019-ncov\%2Fcommunity\%2Fhome\%2Fcleaning-disinfection.html

9. American Academy of Ophthalmology. Alert: important coronavirus updates for ophthalmologists. Disponible en: https://www.aao.org/headline/ alert-important-coronavirus-context

10. Kariwa H, Fujii N, Takashima I. Inactivation of SARS coronavirus by means of povidone-iodine, physical conditions and chemical reagents. Dermatology. 2006;212(Suppl 1):119-23.

11. Sharma N, Khamar P, Sachdev MS, Maheshwari S, Gupta K, Sinha R, et al. All India Ophthalmological Society - Preferred practice in refractive surgery during the COVID-19 pandemic. Indian $\mathrm{J}$ Ophthalmol. 2020;68:1263-8

12. Taravella MJ, Viega J, Luiszer F, Drexler J, Blackburn P, Hovland P, et al. Respirable particles in the excimer laser plume. J Cataract Refract Surg. 2001;27:604-7.

13. Andersen $B M$, Bånrud $H$, Bøe $E$, Bjordal $O$, Drangsholt $F$. Comparison of UV-C light and chemicals for disinfection of surfaces in hospital isolation units. Infect Control Hosp Epidemiol. 2006;27:729-34. 